\title{
The Hydraulic Infinite Linear Actuator with Multiple Rods
}

\author{
Magnus Landberg, Magnus Sethson*, Petter Krus* \\ Saab Aeronautics, Linköping, Sweden \\ E-mail: magnus.landberg@saabgroup.com, magnus.sethson@liu.se, petter.krus@liu.se \\ * Division of Fluid and Mechatronic Systems; \\ Department of Management and Engineering, \\ Linköping University, Linköping, Sweden
}

\begin{abstract}
Future hydraulic actuation systems can be significantly improved by utilisation of a new type of hydraulic linear actuator technology. By enabling multiple actuating elements, integrated in a compact and lightweight single unit controlled by ordinary directional valves it is possible to achieve a high position accuracy. This is a new way to generate and distribute mechanical linear movement and force by using hydraulic actuators in a cost effective way, in less responsive closed loop systems. This is a variant on the Hydraulic Infinite Linear Actuator, HILA, invention. The technology also represents a new sort of digital hydraulics. The presented technology provides typical hydraulic actuator characteristics with high system pressure and potentially no external oil leakage. It is based on a well-known hydraulic clamping element technology used in a new way, where the piston and the piston rod can quickly be coupled and uncoupled by means of the clamping element in a failsafe way by using well defined incremental steps. HILA in its simplest usage, provides new features to hydraulic cylinders such as providing very long strokes, high rod speed, and small chamber volumes which means high stiffness and low capacitance. The aim of this study is to present a fundamentally new way of using hydraulic actuators. The invention is called the Hydraulic Infinite Linear Actuator with Multiple Rods, HILA MR. The study presents the idea, principles and feasible combinations of the technology. Also applications in the aircraft and robotic field will be presented.
\end{abstract}

Keywords: hydraulic actuator, incremental control, snake-like robot, robot arm

\section{Introduction}

Linear hydraulic actuators is highly competitive, unique in some aspects and have significant advantages compared with electric motor drives and electric actuators. These advantages are higher power to weight ratio, stiffer than electric drives, smoother performance at low speed, wider speed range, and greater extent of self-cooling even in stall condition [1].

In areas where high forces and precise movements are needed, hydraulic solutions are often required. Reducing the size of fluid power components and systems, by increasing power density, while maintaining power output provides a great benefit. There is a need of multiple actuator functions with high requirements for strength and stiffness to be implemented especially in confined working spaces. These are common in the aircraft and automotive production, where also compactness and agility are advantageous. These requirements are also common in robot applications, such as articulated robots and snake-like robots.

Additionally, the hydraulic cylinder needs to be designed for longer strokes for use in Cartesian/Gantry robots, tooling machines and additive manufacturing of bigger objects.

The basic invention, in this paper, is a hydraulic cylinder with a releasable piston. It is called the Hydraulic Infinite Linear Actuator (HILA) and is presented in [1]. A hydraulic membrane, the clamping mechanism, is connecting the piston and the rod when pressurised. By using simple logic valves for pressurizing, the piston and rod can be connected and disconnected with maximum secure and reliable clamping in a fast way.

The paper presents a new way of using hydraulic actuators and reports the present state of the HILA development project, that is a collaborative work between Linköping University and Saab AB. In this paper, the focus will be on
Non-reviewed paper. finding interesting applications that can further enhance the development process of the project. Hopefully, at the same time, the study of the applications will have commercial impact. The ideas and principles of the multiple rod technology will be presented, mainly on a conceptual level. To some extent this project takes the hydraulic cylinder technology into new exciting areas, as this technology has been developed slowly in recent decades compared to electric linear actuators.

The disposition of the article is as follows. It is divided into four main parts, see fig 1 . The first part is a description of the basic HILA technology with only one piston rod; the structure and its operation of a standard cylinder. It also describes how bronze bearings and boots can replace the rubber seals between the piston and the cylinder; the advantages and disadvantages, including removing of external leakage, the ability to handle lateral forces and increase the maximum rod speed.

In the second part, HILA with incremental control is presented. It adds new features to present hydraulic cylinder technology and can be a potential alternative to expensive electric and hydraulic servo systems, when high position accuracy is the primary goal within less responsive closed loop systems. The technology also represents a new sort of digital hydraulics.

An aircraft high lift flap system with trim capability has been chosen as a suitable example for aviation applications. This normally requires expensive, complex and maintenance intensive electrical or hydraulic actuation systems. The presented technology can also be used on the leading edge slat system. 


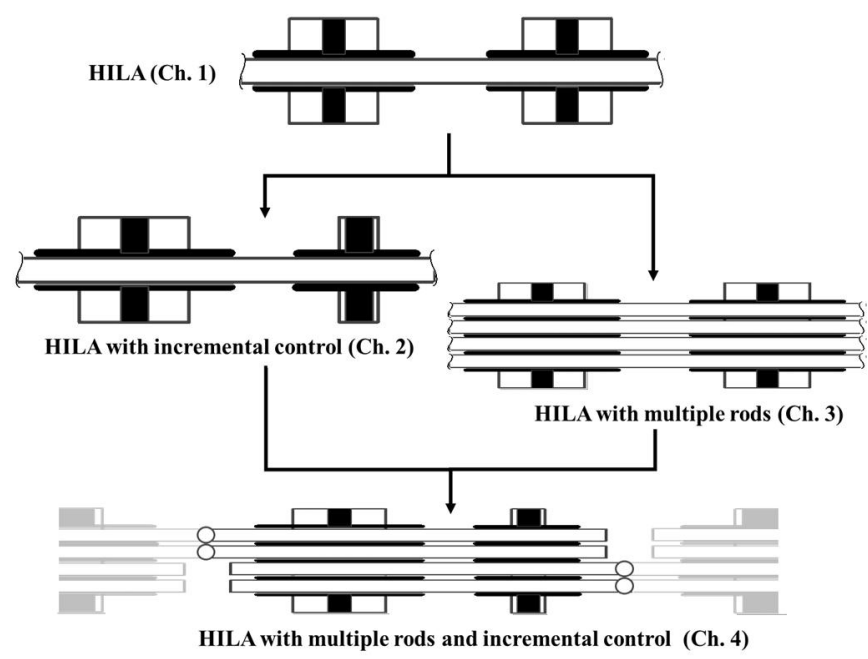

Figure 1. The disposition of the paper, chapter 1 - 4 (Grey parts are surrounding machine elements).

The third part introduces HILA with Multiple Rods (HILA MR) technology. It enables a completely new way to generate and distribute mechanical linear movement and force by using hydraulic actuators, maintaining high pressure, compactness and energy efficiency. An unmanned aircraft, which flies autonomously has been selected as another suitable example for aviation control application. It is also described how a single position sensor on the common piston can be used to determine the absolute position of the individual piston rod.

In the fourth part HILA MR technology is combined with incremental control using ordinary directional valves. It is applied to robot arm segments. By manoeuvring four piston rods simultaneously, twin rotary actuation systems for pitch and yaw in each robot arm segments end is made possible. With large and small incremental steps executed in two linked robot arm segments, it will be able to have a more accurate and time efficient method for angle positioning. The segments can be built together in series creating a snake-like robot. Such high agility robot configuration is capable of working in confined spaces and reach the farthest ends of the cavities of for example an aircraft wing tank. This is an aviation manufacturing example.

\subsection{Introduction to HILA technology applied on one piston rod}

The basic actuator innovation, the Hydraulic Infinite Linear Actuator (HILA) is presented in more detail in [2]. In its simplest usage it is characterised by providing very long strokes, high system pressure, power efficiency, compactness and small chamber volumes. The actuator has a higher stiffness and higher natural frequency compared to conventional hydraulic cylinders. These factors are favourable in control design [2]. The higher system pressure and symmetric piston areas allows for an even more compact system design, with lower flow levels and a smaller reservoir.

The basic invention is a hydraulic cylinder with a releasable piston. A hydraulic membrane, the clamping mechanism, is connecting the piston and the rod, when pressurised. By using simple logic valves for pressurizing, the piston and rod can be connected and disconnected with maximum secure and reliable clamping in a fast way (see fig. 1).

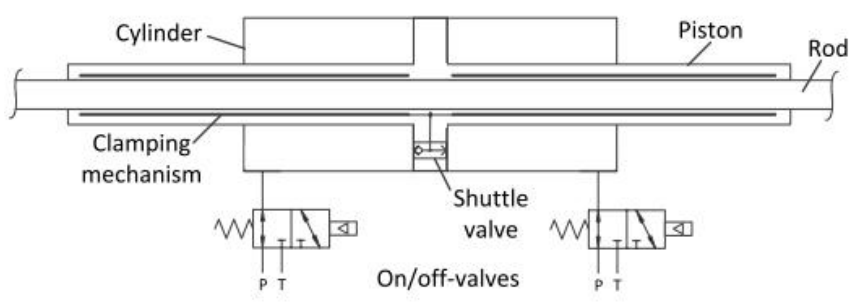

Figure 2. Hydraulic cylinder with a releasable piston.

In the concept in fig. 2 the cylinder chamber with highest pressure pressurizes the hydraulic clamping element using a shuttle valve. The clamping element's surface against the rod is large, which means that the clamping element and the working piston is partially outside the cylinder. The linear hydraulic actuator consists of two double acting cylinders with a common rod in one variant (see fig. 3). For movements one of the pistons is connected alternatively to the rod providing the drive. In this way the two pistons are moving the rod alternatingly in a kind of rope climbing motion.

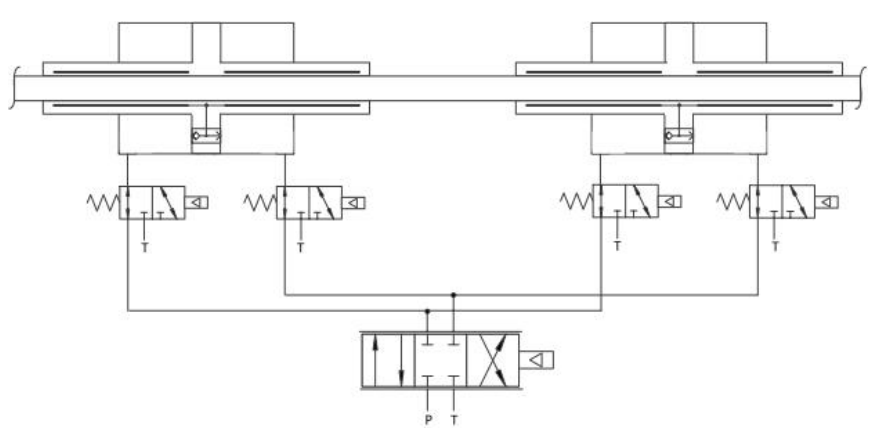

Figure 3. An example of implementation of HILA actuator.

In fig. 4 the actuation process of the cylinders is described. After step 7 the process will continue with step 2 (see also https://www.youtube.com/watch?v=tVJkqC2w5ws ). 


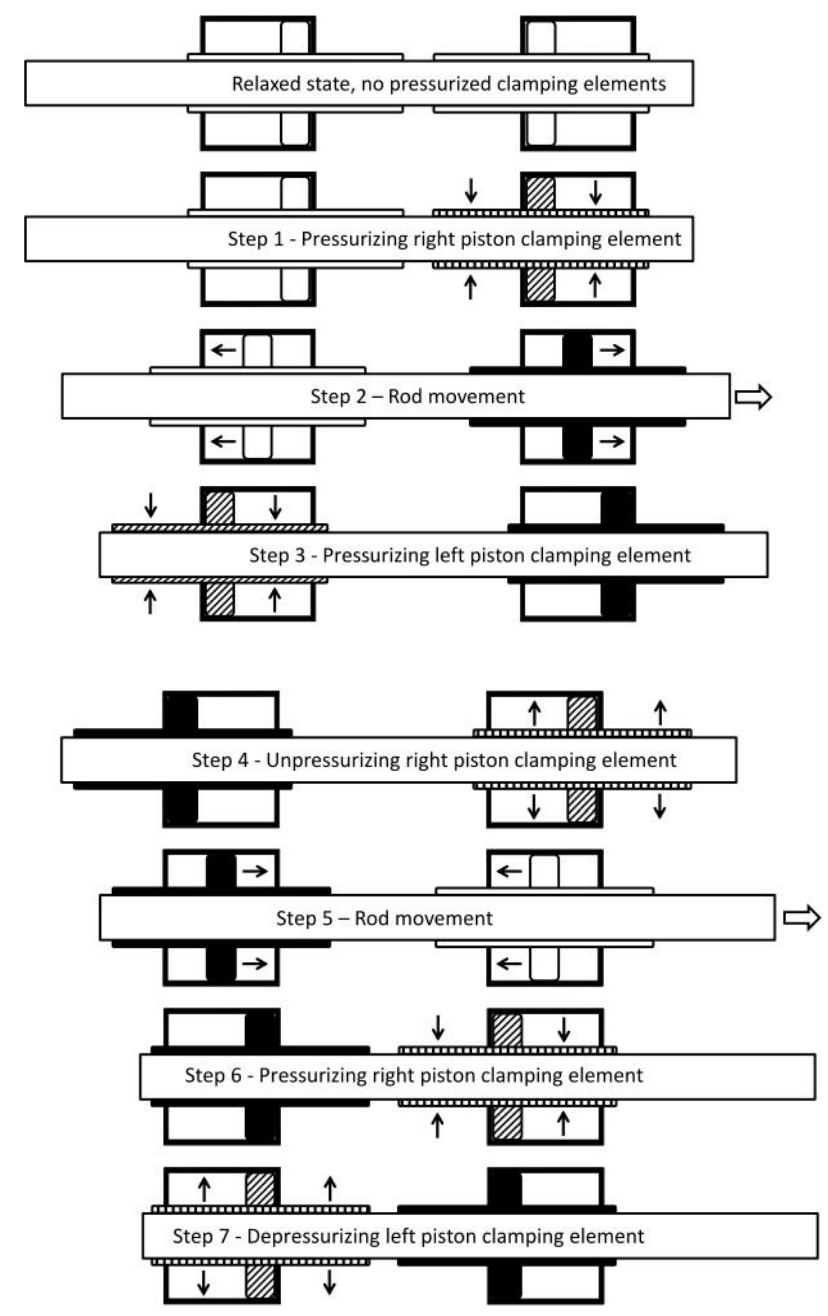

Figure 4. The actuation process of the cylinders.

A parallel to the novel hydraulic actuator is the inchworm motor. This is a device that uses piezoelectric actuators to move a shaft [3].

\subsection{Separate pressurisation}

An identified problem within this HILA design is unintended clamping when the piston is in retracting mode. The pressure level must be kept rather low in the cylinder chambers during retraction in order to avoid inadvertent self-clamping [4].

A solution of this problem is to separate the pressure supply to the clamping element. The pressurization of the clamping element and thus engaging can be implemented by a separate port $C$ through the piston (see fig. 5) and using a separate $3 / 2$ valve. The purpose is to pressurise the small membrane volume and a very small flow is needed and thus a small valve can be used.

The separate pressurisation opens up for a new degree of freedom in control. The piston and the clamping element can be independently controlled in time. The clamping element can be pressurised before or after the piston is pressurised. This solution means also that the shuttle valve in the piston can be replaced.
With this arrangement, full system pressure can be obtained and maximum performance in the clamping element between the piston and the piston rod at valve opening, regardless of the current pressure in the cylinder chambers or load case. This facilitate to minimize any micro slip between the piston rod and the piston, during the clamping and unclamping phase. Another advantage is that inadvertent self-clamping can be avoided.

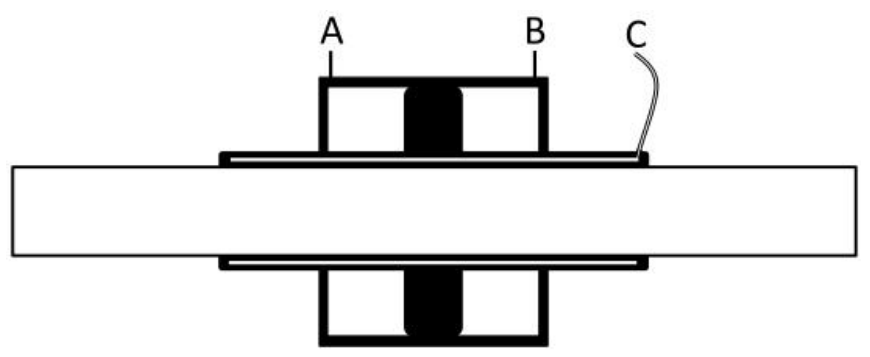

Figure 5. Separate pressurisation of the clamping element.

\section{HILA with incremental control}

The two predominant forms of linear motion control is hydraulic cylinders and electric linear actuators. Electric actuators are often selected as they provide a higher flexibility regarding the motion-control capabilities compared to hydraulic cylinders with directional valves, regarding position, velocity, acceleration and repeatability [5].

A conventional hydraulic cylinder, controlled by ordinary directional valves, works well for end-to-end position applications. A mid-stroke positioning application is more demanding, and requires a more complex control valve solution. Mid-stroke positioning for hydraulic with directional valves is an open-loop operation which requires manually operation.

More advanced hydraulic servo hydraulic systems, provides more accurately control position, velocity, and force, but they require a servo controller, an electrohydraulic servo valve, and a position feedback system. They also add complexity and cost to hydraulic systems and they are sensitive to contamination [5]. New digital hydraulic technology has been introduced in recent years in order to overcome this problems.

\subsection{Digital Hydraulics}

Digital hydraulics or digital fluid power is a broad research field and several research institutes and companies contribute the research. The technology offers several new ways to implement highly efficient hydraulic systems. New applications are emerging and several branches exists, within following areas; valves, pumps, actuators and transformers. Digital fluid power could be defined as follows: "Digital Fluid Power means hydraulic and pneumatic systems having discrete valued component(s) actively controlling system output" [6]. There are two main branches of digital fluid power; systems based on switching technologies and systems based on parallel connection. Both can be applied in several different ways. 


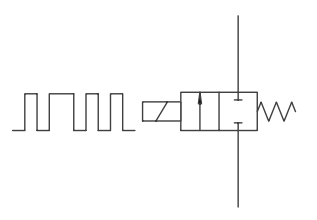

(A)

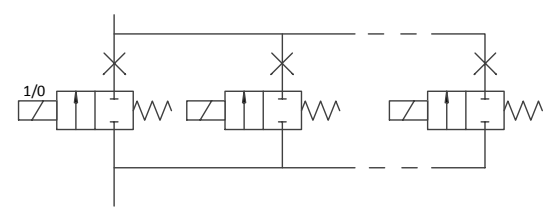

(B)
Figure 6. Systems based on switching technologies (A) and systems based on parallel connection (B).

The switching technology can be implemented as shown in fig. 6A by a switching controlled two-way valve. The valve controls the average flow area by the high frequency modulation and pulse-width modulation technology (PWM) is often used. The other way is to implement valves in parallel as shown in fig. 6B. The total flow area is the sum of the flow areas of the open valves. The main difference between the methods is that the parallel valves do not need switching to maintain a determined flow [6].

Connecting several on/off valves in parallel to form a Digital Flow Control Unit (DFCU), which is the analogue of a DAconverter, as shown in fig. 6B. This approach, needs more valves, but valves can be simpler and with a slower response. The approach gives also other benefits, good motion control with slow-response valves, improved redundancy and reduced durability requirement, when compared to PWM methods. Characteristic of digital hydraulics is that it can use standardized fast, robust and simple valves. They are insensitive for contamination and possibly zero leak. This combined with a high degree of flexibility and programmability opens up for many new design solutions. The drawbacks includes pressure pulsations and noise, durability and life time with switching technology, physical size and price with parallel connection technology and finally complicated control [6].

\subsection{HILA with incremental control}

One combination of HILA technology comprises a HILA cylinder which allows moving of the piston rod in combination with a static clamping unit. The piston rod movement is performed in discrete, incremental steps, like a bang-bang control strategy in a digital hydraulic system. For a feeding cylinder an end position cushioning is needed. End position cushioning ensures a controlled deceleration of the stroke velocity in both end positions. This means that the actuator will not go into hard ends. This cushions eliminates stress on the actuator elements and increase durability. The movements will be smoother.

The operating schedule is as shown in fig. 7. Ordinary directional valves are used for controlling the flow. The clamping elements in the static and moving element takes turn to keep the piston rod during the movement. This positioning technology offers a cost-effective, compact, simple and robust way to move a payload linearly from point A to point B.

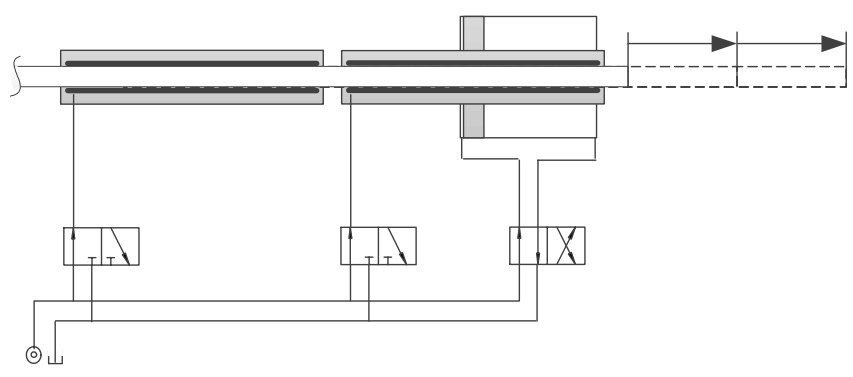

Figure 7. Incremental motion with a HILA cylinder in combination with a static clamping unit.

\subsection{Adding fine tuning capability}

The displacement of the load occurs in discrete steps. This means little opportunity for accurate control. Opportunities to correct and fine-tune the position is missing. But can easily be achieved by a slight modification of the design to obtain a higher positioning accuracy. The static clamping unit in the concept can be relatively easily modified. It can be replaced with a HILA cylinder with a considerably shorter stroke than the other HILA cylinder. The short stroke cylinder is very useful for enabling position fine tuning. With small corrective steps a high positioning accuracy is enabled without expensive hydraulic valve solutions, see fig. 8 .

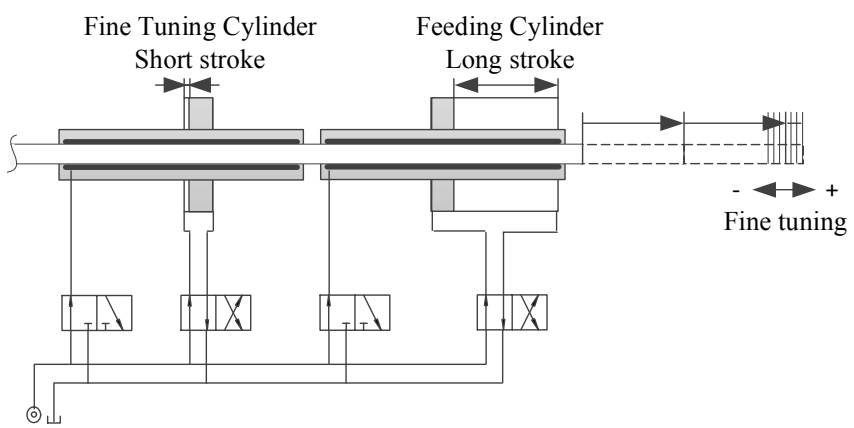

Figure 8. Incremental motion with a HILA feeding cylinder in combination with a fine tuning HILA cylinder.

The characteristic feature of this design is that the two cylinders HILA change function during the different phases. In the Discrete Feeding Mode the fine tuning cylinder acts as a static clamping unit and the feeding cylinder is moving with incremental motion. In the Fine Tuning Mode the roles are shifted, as shown in the fig. 9. The fine tuning can be done in both directions. And finally, when the required position is reached, both cylinders enters the static clamping mode, providing high stiffness. 

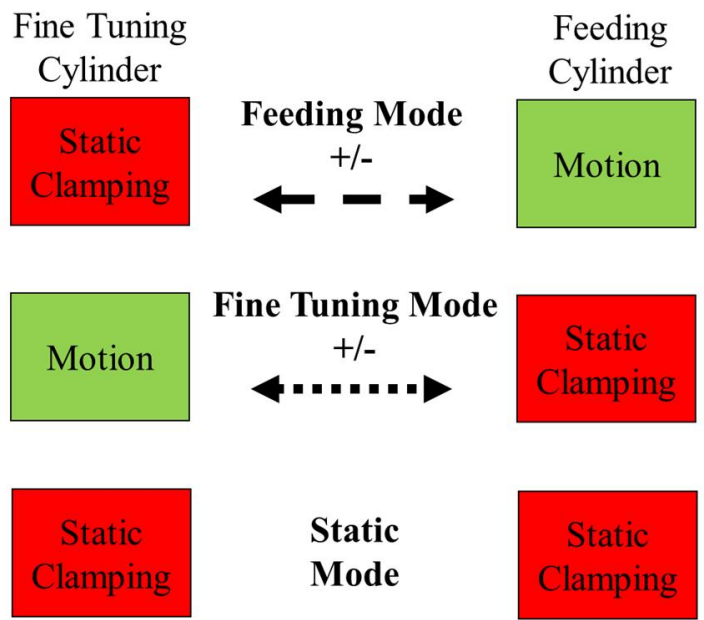

\section{Static \\ Mode}

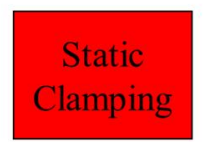

Figure 9. Discrete feeding, Fine Tuning and Static Mode.

In many actuation applications, a more advanced control system with expensive servo valves or proportional valves, is not a viable option because of the high cost associated with this type of valve technology. With the proposed concept, a system with high positional accuracy can be obtained in less responsive closed loop systems.

The proposed HILA with incremental control adds new features to present hydraulic cylinder technology and can be a potential alternative to expensive electric and hydraulic servo system, when high position accuracy is the primary goal. The technology also represents a new sort of digital hydraulics. The 3/2-valve may be implemented in the form of two on/off valves.

The needed space for a HILA system with incremental control can be smaller than for a conventional hydraulic systems as a higher system pressure and smaller reservoir is possible. A HILA system can generate 333high forces as it uses high system pressure. The present HILA technology allows pressures up to $45 \mathrm{MPa}$ [7].

A HILA actuator system can advantageously be built together with a hydraulic supply including an electric motor and a high pressure hydraulic pump to a very compact system at the local site where the actuator is to be installed. And the power can be transmitted using an electrical cable instead of a long hydraulic hose or pipe. In areas where sensitive servo or proportional valves and feedback systems not are suitable, such as agricultural hydraulics, HILA with incremental control fine tuning provides a robust alternative.

\subsection{Application Example - High lift systems}

HILA with incremental control with fine tuning is also transferable to an aircraft context controlling surfaces. Examples of applications where positioning speed is of minor importance are secondary control surfaces, as flaps and slats on high lift systems in aircrafts.

To implement the digital hydraulic technology in an aircraft context means special adaptation must be done to deal with the pressure peaks generated in the valves which are connected to the fatigue related issues in cylinders and pipes.
It is important to slow down the valve closing time in order to provide reasonable pressure peak levels for fatigue reasons.

\subsubsection{Introduction to high lift systems}

A high-lift device is a component or mechanism on an aircraft's wing that increases the amount of lift produced by the wing at start and landing, by increasing the wing area. Common movable high-lift devices include slats on the leading edge and flaps on trailing edge of the wing. Today's commercial airplanes are equipped with high lift devices to provide lift augmentation at low speed during take-off and landing as the wing design is optimised for the cruise speed regime. In order to keep take-off and landing speeds within reasonable limits, powerful high lift devices is required [8].

State-of-the-art high lift actuation systems often consist of a mechanical transmission shaft system (that transmits the mechanical energy from a central motor to the rotary or ball screw actuators, which are located along the transmission shaft and move the high lift surfaces [8].

This architecture assures a synchronous deployment of all flap and respectively slat panels but prohibit functional flexibility. The optimisation capabilities of today's highly optimised high lift system architectures has reached a plateau and are limited to small local improvements [8].

An advanced high lift actuation system architecture with distributed active controlled flap actuators offers the capability for implementation of additional functionalities for the trailing edge with benefits on aircraft level and simplifies manufacturing [8].

HILA actuator with incremental control with relatively simple means can be adapted for a high-lift flap system. Here it is proposed a fully independent HILA High Lift actuation system for flaps is similar to HISYS A350 XWB's high lift system concept, described in [8], but is built with HILA actuators at each drive station. The hydraulic actuators do not need dedicated power electronics. In fig. 10 two HILA actuators are installed in pairs on each inboard and outboard flap panels on left and right wing.

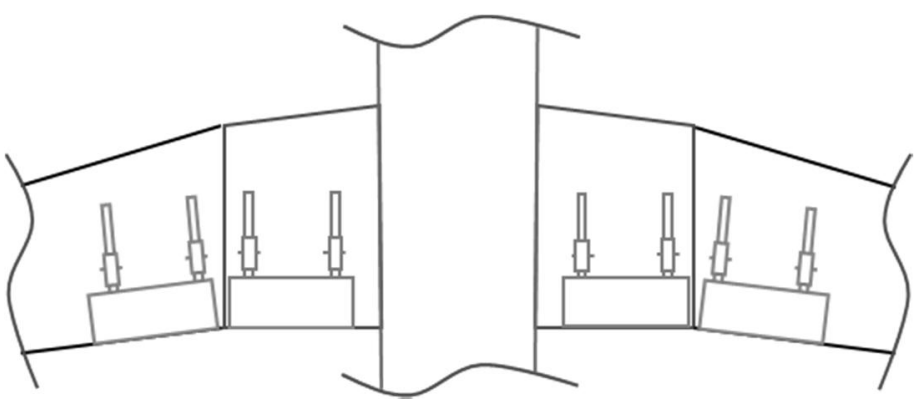

Figure 10. HILA High Lift actuation system on inner and outer flap on left and right wing.

The HISYS concept provides an advanced high lift actuation system architecture with distributed active controlled flap actuators offering the capability for implementation of additional functionalities for the trailing edge with benefits on aircraft level and improvements regarding manufacturing and assembling. Depending on the flap setting, the computer- 
controlled spoiler automatically moves into the most efficient position during cruise flight. Drag can be reduced by up to two per cent at high gross weights, resulting in considerable fuel economies. Weight savings on the order of half a tonne for the wing box are feasible by using differential flap settings to alleviate loads by changing the centre of lift for loads management [8]. HILA concept also has the same functional benefits and reduced manufacturing effort as the HISYS concept.

\subsection{Requirements on a high lift hydraulic actuator}

The requirements that characterize a hydraulic actuator for a high-lift flap applications are as follows:

- High demands on position accuracy

- Relatively high air loads, but no mass inertia

- High stiffness

- Low operating actuator speed

- Lightweight and compact design

- High reliability, a failsafe solution and a high ability to detect errors

- Non-complex control algorithm

- No high pressure peaks during operation. Soft start and stop of cylinder manoeuvring.

- Parallel and synchronous movement of the actuators

- Low temperature

\subsection{HILA High Lift System}

The proposed high lift system with differential flap setting, enables optimization of the cruise aerodynamic efficiency and loads through the control of the wing centre of the lift position by differentiating the inner and outer flap. The proposed flap system can be actuated by an electric driven system with ball-screws or a HILA High Lift system, as showed in fig. 11, fig. 12A and 12B.

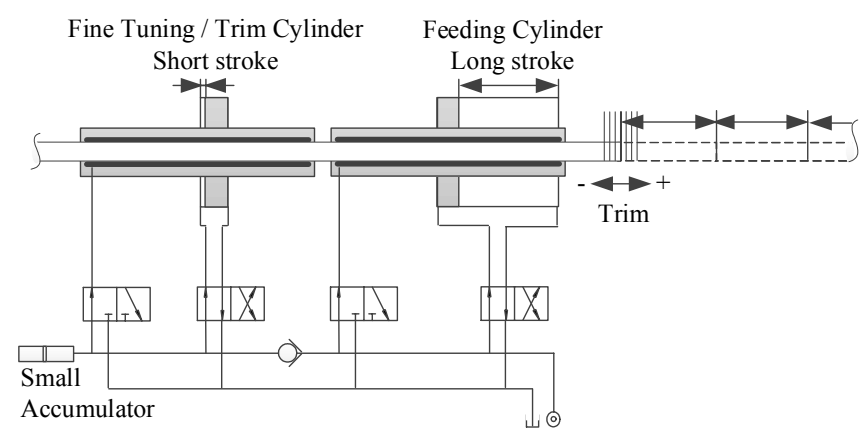

Figure 11. Incremental motion with a feeding HILA cylinder in combination with a fine tuning/trim HILA cylinder.

With HILA technology flap movement is made during takeoff and landing in discrete incremental steps of 2-4 degrees, extending the wing area, as described in previously chapters, with simple, proven and robust ordinary on/off and directional valves. In cruise mode the technology enables trim capability with small incremental steps for fuel saving and load optimization using the fine tuning cylinder.

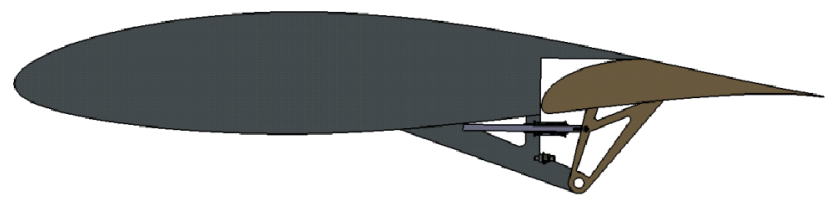

(A)

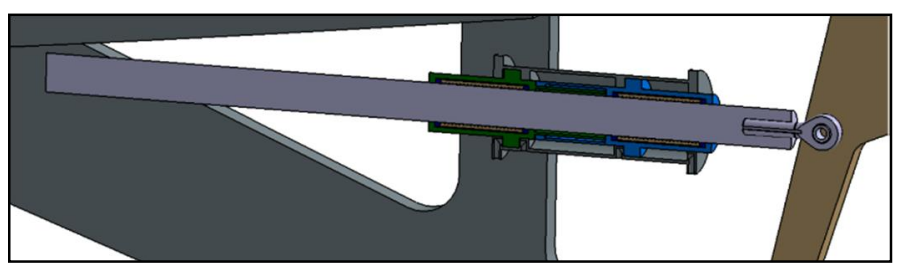

(B)

Figure 12. HILA cylinder installed between the wing and the high lift flap surface.

The feature of this solution is that the compact and leak-free cylinders combined with well-known clamping technology is used. Further a simplified wing design is provided, no rotating and bending shafts along the wing spar are required.

Furthermore, the concept is relatively simple to monitoring with detectable faults. At every start a Pre-Flight Built In Test is performed.

The system provides fail-safe position upon failure of the hydraulic and/or electric power, and at flap asymmetries. The maintenance of the hydraulic system are less demanding and less frequent in comparison with electric ball screw, which is important from a LCC perspective.

With well-known clamping technology and incremental control HILA innovation provides a new, robust and costeffective high lift flap system that is simple in its design and provides both high-lift at start and landing also trim of flap position in cruise mode. This normally requires expensive, complex and maintenance intensive electrical or hydraulic actuation systems. HILA High Lift actuation technology can also be used on the leading edge slat system.

\subsection{Redundancy with HILA technology}

There are a number of fault cases that are handled in a failsafe way with sufficient redundancy in the actuator system and on a system level with HILA technology.

\subsection{Loss of hydraulic and / or electric power}

To handle faults the design has been supplemented with a small accumulator and a check valve, see fig. 11. In the event of loss of hydraulic and/or electrical power, hydraulic pressure on the static clamping element can be maintained by means of a small accumulator and a check valve.

\subsection{Asymmetry and jamming}

To ensure that the two systems HILA on respective flap panel are working in parallel and synchronously there are different 
ways to detect failures together with a position sensor on each actuator.

A simple solution is provided by proximity switches at the end positions of the pistons. If the switching in the proximity switches in the right and left system do not occur simultaneously (some tenths of ms can be tolerated) a monitor detect the motion asymmetry between right and left system.

Another way to ensure the fault occurs, is to compare the hydraulic pressure in the cylinder chambers in the left and right actuator during motion. If the pressure differs too much from each other means that different speeds in the respective cylinder and a risk of jamming exists. A frozen state will be commanded and the flap panel remains in its fixed position throughout the remaining flight.

\subsection{Hydraulic system failure}

Normally a flight hydraulic system has two independent hydraulic systems (HS1 and HS2). The high lift system is supplied with hydraulic power from one hydraulic system, here HS1. In the event of failure of HS1, HS2 is commanded to supply the system through an emergency valve and the shuttle valve, according to fig. 13 .

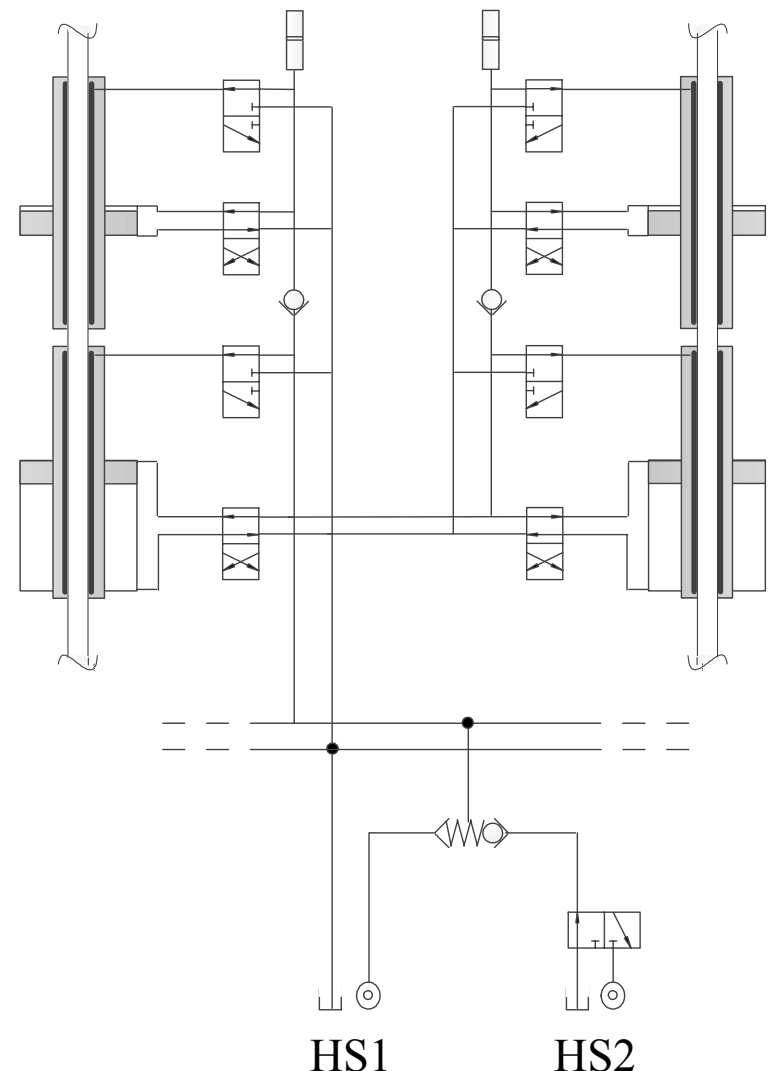

Figure 13. HILA High Lift flap system with left and right actuators, supplied normally from HS1 and in emergency case from HS2.

\subsection{Pressure peaks}

It is important to control the directional valves, included in HILA High Lift system, avoiding pressure peaks and cavitation during the stopping. It can be done by using a suitable opening area gradient in the directional valve. But it can also be combined with end position cushioning. End position cushioning ensures a controlled deceleration of the stroke velocity in both end positions. This means that the actuator will not "bang" into hard-stops or jolt. This cushions eliminates stress on the actuator elements. The movements can be smoother.

\section{HILA technology with multiple rods}

\subsection{Controlling multiple rods in the same common piston}

The separate pressurization of the clamping element opens up the possibility to control the movement of multiple individual linear piston rods in the same common piston.

Here it is proposed a solution in which multiple piston rods are using the same piston. A common hydraulic piston transfer simultaneous movement to a number of piston rods. Each piston rod is connected individually to the common pistons with individual clamping elements or of individual static clamping member by means of individual logic valves (see fig. 14 and 15). A common servo valve or directional valve controls the common piston.

Why gather a number of piston rods in the same piston? This way of arranging actuator movement has advantages but also present some limitations, among others regarding controllability.

In following chapter a number of ways of using this technology in different applications will be presented.

The technology is called HILA with Multiple Rods (HILA MR). HILA MR enables a completely new way to generate and distribute mechanical linear movement and force by using hydraulic actuators.
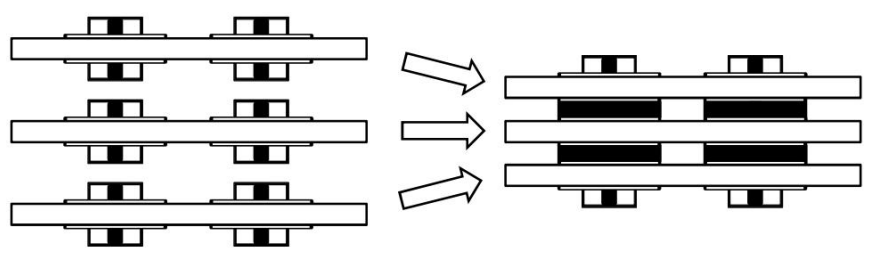

Figure 14. Six individual pistons integrated in two common pistons.

In areas where there are a great need for compact and energyefficient drive units for multiple linear actuation are required HILA MR technology can be a suitable alternative. HILA MR enables a very compact, lightweight and cost-effective multiple actuation, especially if high hydraulic pressure can be used. 


\subsection{Ability to maintain static load for long time}

Multiple static clamping elements integrated in common pistons together can individually perform piston rod movements in different directions. Combined with an optional static clamping element, this arrangement also has an ability to keep a high static load for a long period with almost no energy consumption. Equivalent solution for electric actuators, which do not use self-locking gears such as screwballs, requires a constant supply of current to hold the load.

In some applications, a static clamping in combination with HILA MR technology is very useful. With a static clamping, for example in a robot hand or gripper application, quick and precise positioning with low forces can be combined with high static forces for a long time. In order to control multiple rods with different requirements regarding movement and rest a static clamping unit is needed, see fig. 15 below. The static clamping element provides a parking mode for pistons rod which not are in motion.

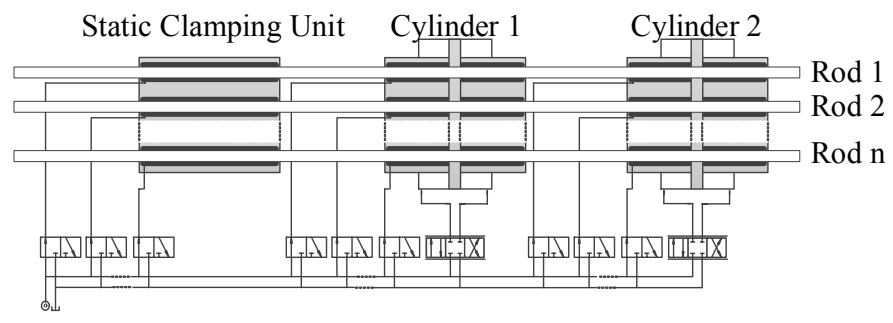

Figure 15. Hydraulic schematic of HILA MR

\subsection{Separate pressurization with micro-valves}

A 3/2 micro-valve controls the pressurization of the respective piston rod clamping elements. It has a switching time of a few milliseconds. Small valves with low power consumption and low flow capacity can be used because the membrane in the clamping element needs a very small volume change during pressurization and clamping.

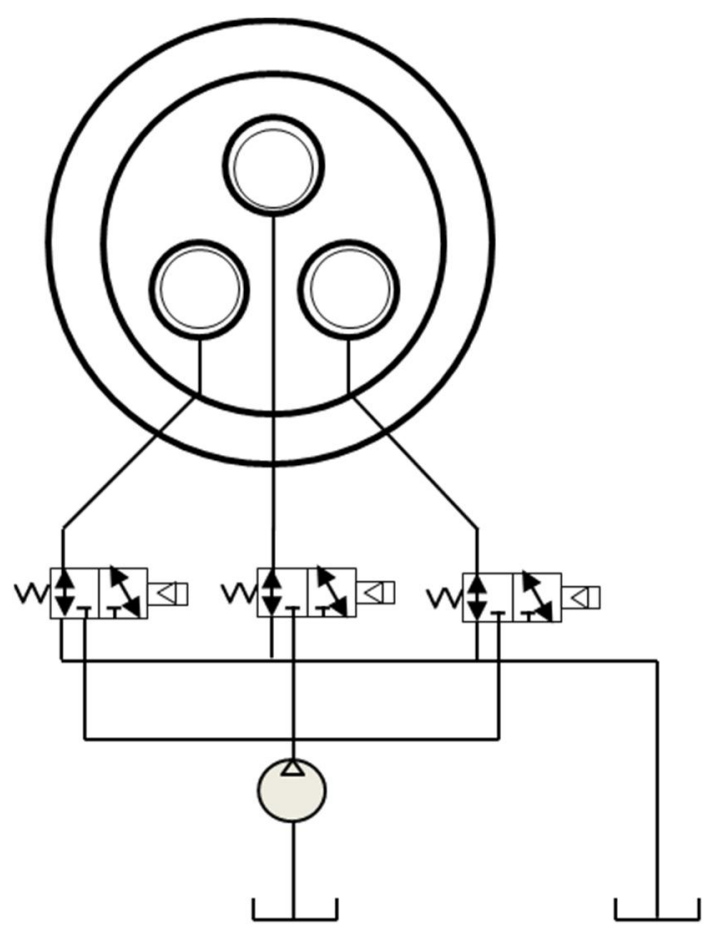

Figure 16. Individual pressurisation of each clamping element in common piston with micro-valves

With a small electric control current in the micro-valve, a large axial force in the piston can be controlled in a simple way (see fig. 16). A large mechanical force in the individual piston rod can be controlled by the micro-valve in a manner similar to how a small gate current in an electrical transistor can control a much larger current. Micro-valves may be placed as an extension of the part of the protruding piston, outside the cylinder. The supply of hydraulic power to the valves is done via a common thin hose and a thin electric multi cable. In order to decrease the clamping element area a higher control pressure may be used. The used clamping technology allows clamping pressures up to $45 \mathrm{MPa}$ [7]. The $3 / 2$ micro-valve may be implemented in the form of two on/off valves.

\subsection{Thermal Handling}

A well-known problem with electric actuators is that they cannot permanently be hold in a position to keep a high load, because they soon become detrimental warm and at the same time as they consume a lot of electric energy (if they do not use a self-locking gear). In a hydraulic circuit, the dissipated heat in the oil can be efficiently cooled off in an oil cooler.

HILA MR technology can handle thermal issues much better than comparable electric alternatives, due to the constantly circulating oil. Especially in applications where continuous $24 / 7$ operation is demanded and/or in applications installed in cramped spaces where no cooling baffle fits, e.g. in a robotic limb, hand or gripper. The risk for overheating which can damage the actuator is low as the generated heat energy is taken away by the circulating oil. In an electric actuator, the heat transport to the air take place locally with bulky air baffles and/or fans. 


\subsection{No external leakage and lateral forces}

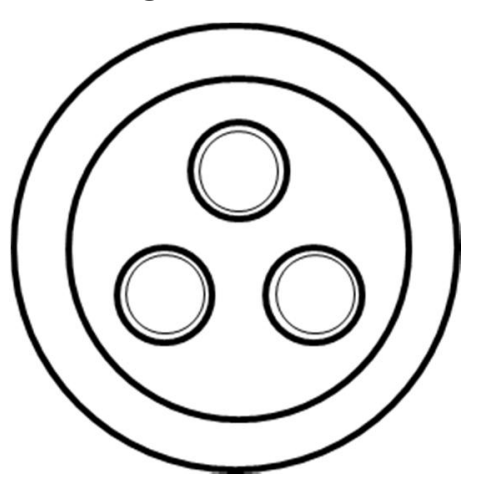

Figure 17. Piston rods not centrally located

The proposed method to locate the rods in the piston, means that all the rods are not centrally located, but distributed radially from the center, see fig. 17 . That means that side lateral forces are generated in the piston if the transferred axial forces in the individual piston rod are not balanced. Lateral forces or side loading is a common cylinder problem that normally leads to premature failure. Lateral forces on a cylinder rod, particularly when the rod is extended, can damage the cylinder and shorten its useful life. Eventually, seals will suffer damage, resulting in external fluid leaks and seal failure.

Lateral forces on the common piston which occurs, can be handled in a non-destructive way by using bronze bearings in a similar way that they are used in hydraulic in-line machines, instead of using rubber sealings.

Introducing bearings, instead of using rubber sealings, provide advantages but also drawbacks, which are solvable. Among them leakage. Hydraulic equipment in general, and hydraulic cylinders especially are leaking oil externally when they are pressurized and work. This is one of the biggest drawbacks in hydraulics and a challenge to handle.

The friction is also higher in a symmetric cylinder than in a conventional asymmetrical cylinder, due to double end sealings. In order to design a power efficient system it is essential to minimize these friction losses and also to achieve better speed performance.

How can the external leakage be minimized? As the piston has a short stroke it is possible to complete the cylinder with boots (rubber or metallic) over the piston. The leaked oil is returned to the reservoir and can be reused instead of contaminating the environment (see fig. 18).

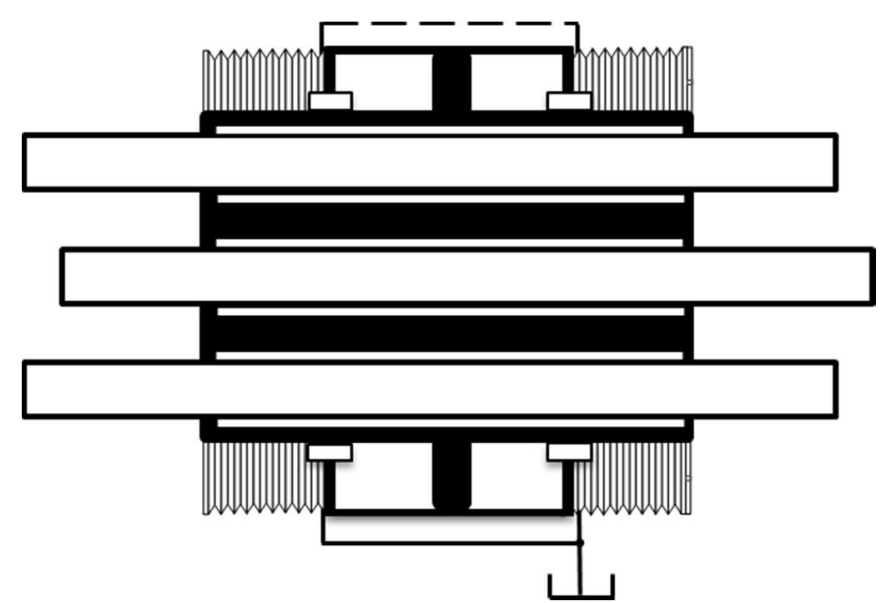

Figure 18. HILA MR with bearings which takes up lateral forces and boots preventing external leakage.

Furthermore, this means that the piston can be kept clean and it does not require a wiper, i.e., no contaminations from the environment enters through the cylinder rod and the seal into the oil chambers when the cylinder is working, which is common in many applications.

The piston can be kept clean but also the oil in the cylinder chambers. The relatively large external leakage that occurs over the seals and which constitute the lion's share of a hydraulic cylinder oil leak collected in the boot and implemented through a connecting hose back to reservoir. It is possible that the wear ring and rod seal between piston and cylinder can be replaced by bronze bearings.

The advantages of this arrangement are several. With the boot a higher leakage of cylinder seals is allowed. This reduces friction and energy losses and the power efficiency of the cylinder is increased. Furthermore, higher rod speeds are permitted when the friction is reduced. This permits in addition that cylinder may be more possible applications, for example for high-speed elevators in high-rise buildings.

Boots solution increases the potential of the cylinder innovation with the risks of oil contamination significantly reduced and higher power efficiency and rod speed can be offered. Though, if the leakage becomes too high the control accuracy can be too low. 
3.6 Common position sensor

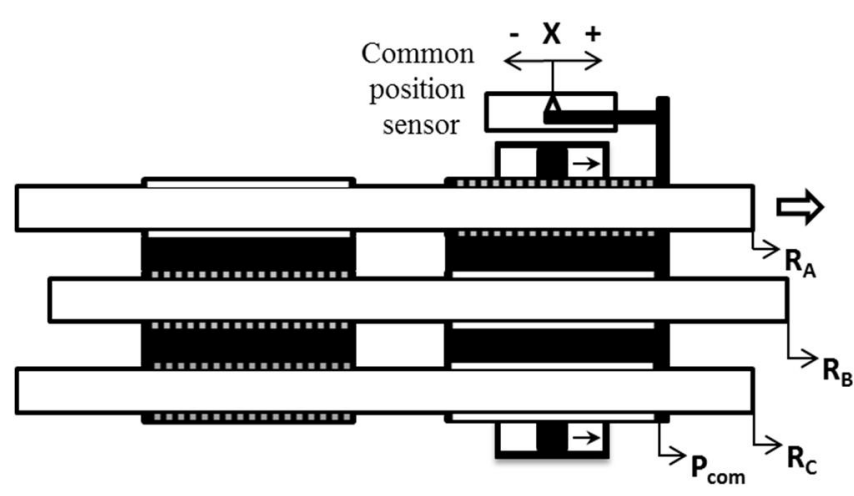

Figure 19. A HILA MR combination with a common static clamping unit and a common cylinder with clamping elements and a position sensor in the moving common piston $\left(P_{\text {com }}\right)$ (dotted line $=$ pressurised clamping elements).

The common piston $\left(\mathrm{P}_{\text {com }}\right)$ that work partially outside the cylinder housing can be equipped with a position sensor for feedback control (see fig. 19). The position sensor can be of different types, e.g. LVDT, potentiometer, etc.

In fig. $19 \operatorname{rod} A\left(R_{A}\right)$ is clamped to the moving common piston $\left(\mathrm{P}_{\text {com }}\right)$. Rod $\mathrm{B}$ and $\mathrm{C}$ are static clamped. The relative motion which is done with rod A clamped to the common piston is calculated.

This relative position information, using an embedded computer, is used to estimate the absolute position for rod A and for each piston rod which is moved. This position information is essential for a control system. The common common position sensor means that a large number of individual position sensors can be eliminated. This arrangement avoids a lot of different failure modes and saves volume, weight and cost.

The position information from the common position sensor must be complemented with different types of position synchronization of the respective piston rod and built-in tests to ensure that the location information for each rod can be trusted at every time. At start up all piston rods can be positioned at a hard stop with a known position and then be synchronized. If not repeated synchronizations are performed you cannot rely on the information from the common position sensor.

It is also of great importance that no micro slip occurs between the rod and the common piston at clamping and unclamping. This risk is minimized if clamping and unclamping takes place when the piston and piston rod is stationary still or have the same speed.

\subsection{Infinite stationary hydraulic stiffness}

The considered concept, HILA MR, allows moving the piston without moving the disengaged rods. Thus when the desired rod positions are reached the disengaged piston can be moved to one of its end stops. After engaging the cylinder and pressurizing the one cylinder chamber to push the piston against the end stop the elasticity of the mechanical contact is dominating the cylinder stiffness, see fig. 20. Thus the hydraulic stiffness becomes infinite. In this consideration the elasticity of all mechanical components like structure and rod are neglected [4].

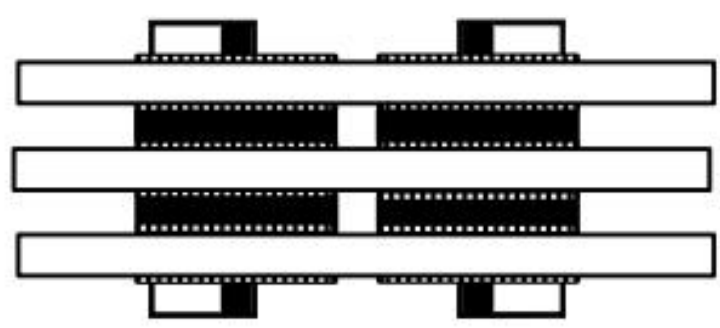

Figure 20. Two cylinders at opposed side end stops, with infinite stiffness.

\subsection{Hydraulic Time Sharing}

HILA MR technology enables time sharing and a load sensing.

By using a time sharing strategy for the individual piston rod movements advantages can be obtained. If a piston rod is moved at a time and the other piston rods are in static mode following advantages is obtained: The common piston area can be reduced. The force of the piston rod having largest force demand determines the common piston area. The common piston area can be reduced and the cylinder can be designed more compact and lightweight. Also the supply system can be designed with lower power requirements.

\subsection{Time sharing hydraulics in an unmanned aircraft}

A suitable field for the proposed time sharing hydraulic technology with HILA MR technology is in unmanned aircrafts.

An aircraft has a number of subsystems containing hydraulic actuators, among others, flight control system, landing gear system and fuel system. A Medium-Altitude Long-Endurance (MALE) unmanned aircraft, which flies autonomously has been selected as a suitable application.

The flight control system can be divided into primary and secondary control surfaces. The primary control surfaces consist of ailerons, elevators and a rudder and its task is to maintain the aircraft's stability at disturbances that occur in flight and control the aircraft in pitch, roll and yaw of a predetermined flight trajectory.

The rudder commands to maintain stability are time-critical and unpredictable due to wind gust and side winds, i.e. they must be executed without delay, and they have the highest priority. Commands for control in pitch, roll and yaw are less time-critical and follows in order of priority. Lower priority has the operation of secondary control surfaces, the flaps for high lift, and the landing gear actuation, which if necessary can be divided into phases

Besides the rudder commands required to maintain stability, the other commands can planned in advance as they are predictable and less or not time-critical. These commands need not to be performed in parallel, but they may be 
commanded in series. This is possible in an UAV when a pilot is not present in the control loop.

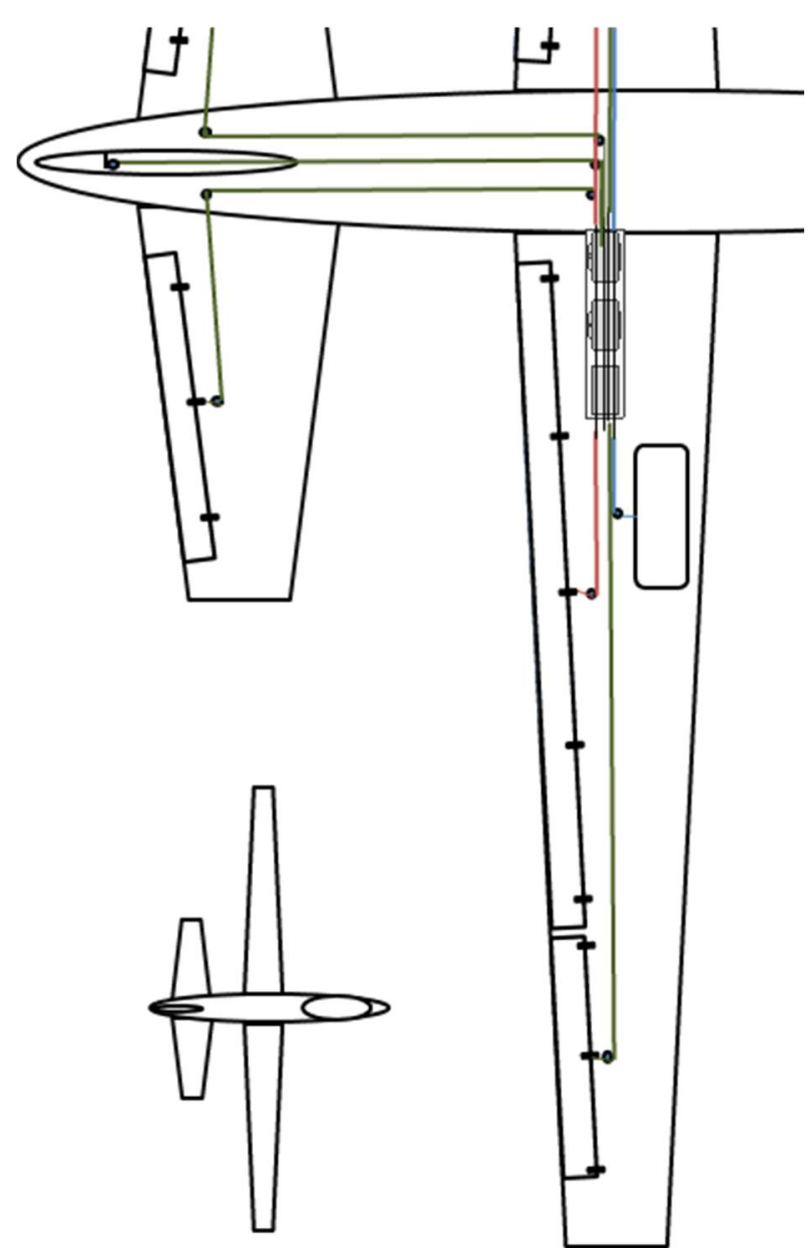

Figure 21. A MALE UAV with HILA MR technology

In most aircrafts there are active rudder activities only during a few percent of a total flight, especially during long distance flights. Under these conditions a time sharing hydraulics system is preferable. Rudder and landing gear commands can be executed in series. In case of a wind disturbance, the secondary control surfaces and/or landing gear manoeuvring can be stopped temporarily, in order to move the primary control surfaces and stabilize the aircraft.

With this conditions, a hydraulic actuator system based on HILA MR technology and a hydraulic supply system can be designed, which has low power demand, is more compact, lighter, more energy efficient, and not least cost-effective. HILA MR technology, can here be used here in combination with wire cables that transfer power and motion to the respective consumer. Wire technology has been used for many decades in aviation, controlling surfaces. See fig. 21.

In the design of hydraulic systems in mobile vehicles, and in particular, in an aircraft, a large focus is on minimizing system weight and energy consumption. In aviation, the demands on system safety is high, but for space reasons it is not described here how the system can be built fault tolerant with redundant solutions.

\section{HILA MR with incremental control}

HILA MR technology can be combined with the incremental control methods introduced in earlier chapters. There are interesting applications for this combination in the robotics field, which will be presented and analysed in this chapter. It will also be conceptually shown that in this field, HILA MR with incremental control enables more accurate and time efficient methods for positioning.

\subsection{Robot applications}

HILA MR technology is particularly suitable for applications where multiple actuator functions with high requirements of strength and stiffness have to be implemented in confined spaces. This requirements is common in robot applications, such as articulated robot and snake-like robots where also a less responsive closed loop system can be used.

\subsection{Description of the base element}

HILA MR technology enables a flexible, compact and rigid actuator, which is highly desirable in design of articulated robots arms or snake-like robots. This is provided by manoeuvring four piston rods simultaneously in a robot arm segment.

An articulated robot arm uses rotary joints to access its work space. Between the joints there are rigid links. The joints and links are arranged in a kinematic chain, so that one joint supports another joint via the link or spacing section in the chain. The terminus of the kinematic chain of the robot arm is called the end effector. As end effector a gripper, a device for grasping or holding a payload, or a tool, is normally used.

The rotary joint between the sections allows rotational motion. The flexibility of robot arms depends of the rotational ability between each section. Most robot arms in an articulated robot only have one axis in a single section, which is equal to one degree of freedom (DOF). In order to get better access to confined spaces access a universal joint is preferable. HILA MR technology enables a joint with two DOF, both in pitch and yaw, see fig. 22, in each center of rotation.

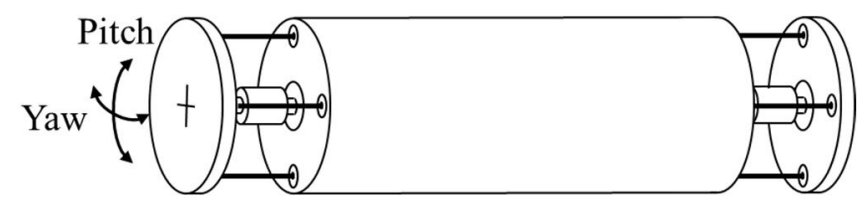

\section{Figure 22. Actuation in pitch and yaw rotational direction} at left and right end of the robot arm segment.

In many applications a robot arm should provide also high accuracy, repeatability, resolution and speed.

The resolution is the smallest increment of motion or distance that can be detected or controlled by the control system of the robot arm. 
The best solution with respect of these requirements is to provide a hydraulic servo valve. It is not always possible to provide a servo valve for control of the respective piston rod because of cost and space reasons.

In some applications with less responsive closed loop systems, but with high resolution and high position accuracy requirements, an incremental control may be a better solution.

Here it is described such a robot arm solution where HILA MR with incremental control is used with a fine tuning cylinder and a feeding cylinder, see fig. 23 .

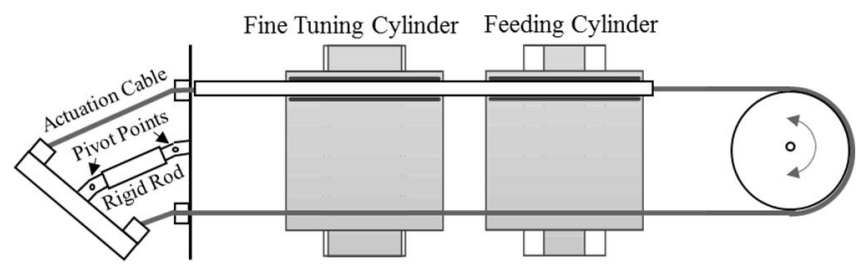

Figure 23. Rotational actuation in a segment of snake-robot arm construction with HILA MR with incremental control.

In order to employ twin rotary actuation system and two DOF in each robot arm segments end and maintain stiffness and compactness a new snake arm robot construction is used described in [10] using stiff actuation cables and wheels to close the force loop.

One cable in a pair of actuation cables pulls a distance and the other cable in this pair extracts the same distance, which causes the bending deformation of the compliant joints and holds the cables of this pair in tension in any kinematic configuration [10]. Here it is proposed that the cable is actuated by HILA technology instead of an angular actuator.

With only two ordinary directional valves and eight small 3/2 valves, four piston rods can be controlled and actuated independently and a high position accuracy can is achievable for each piston rod. These piston rods in turn control the rotation in pitch and yaw in each end of the segment by using cables, according to figure 23 . The hydraulic schematic and piston rods connection to each rotational direction is described in the fig. 24. The kinematics and interaction of the actuation cables, the cable, and the wheel is presented in detail in [10].
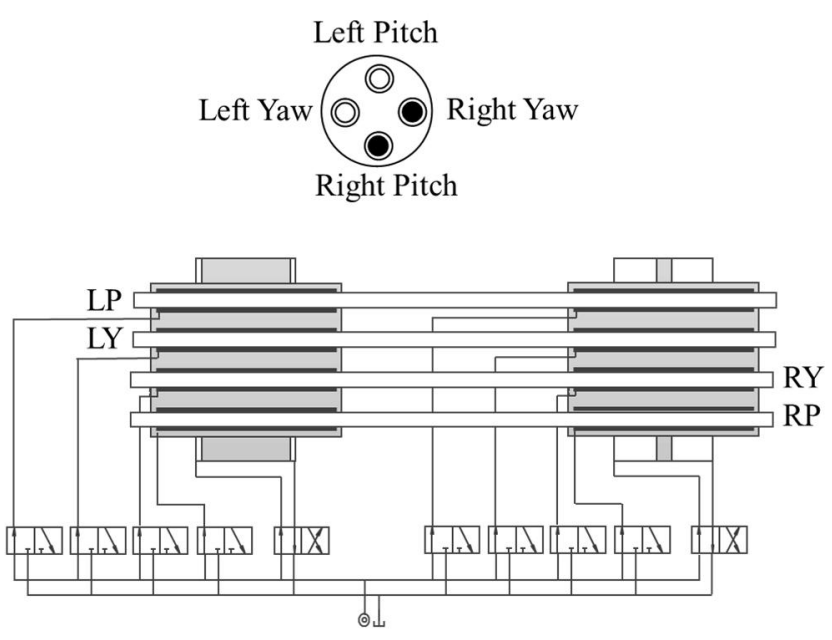

Figure 24. HILA MR hydraulic schematic and piston rods connection to each rotational direction ( $L=$ left, $R=$ right, $P=$ pitch,$Y=y a w)$

\subsection{Incremental control with a piston rod pair}

The same incremental control technique described for HILA applications in earlier chapters where only one rod is used, is preferably used also in this application (see fig. $25 \mathrm{~A}$ ). Rotational actuation is obtained with two segments with HILA MR with incremental control. Angle positioning in pitch and yaw can be implemented with two piston rods, each of which is controlled by the two pistons. An even higher accuracy is achievable with this arrangement, in comparison with the previously presented technology.

The four pistons have unique stroke lengths, i.e. there are four different available strokes for incremental control of the angular position (see fig. $25 \mathrm{~B}$ ). The piston with the shortest stroke (3) determines the resolution ( $r$ ) in the control system, the next piston (2), the stroke of which is $m$ times longer, has an incremental stroke $\mathrm{m} * \mathrm{r}$. The next piston (4) has the stroke $\mathrm{m}^{2} * \mathrm{r}$ and the last piston (4) has the stroke $\mathrm{m}^{3} * \mathrm{r}$. The multiplication factor $\mathrm{m}$ can be varied depending on requirements on total stroke and time requirements, i.e. number of allowed feeding and fine tuning iterations.

The kinematics of the robot arm is described by a chain of skew transformation matrices which represents the relation between the pair of pistons to angular motion of each individual segments and are described in [10]. The largest strokes can be combined with end position cushioning in order to mitigate jerky motion. 


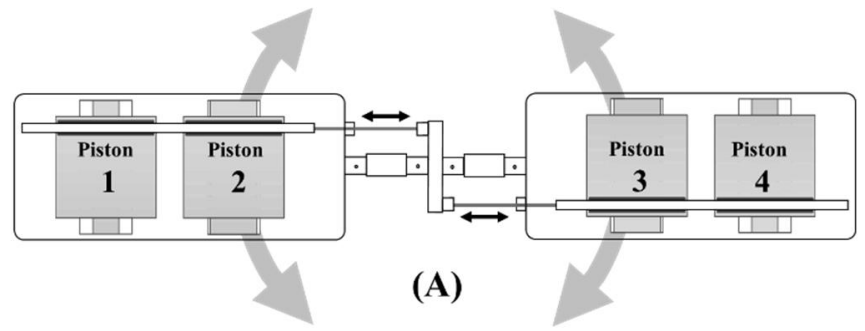

\begin{tabular}{|c|c|c|c|c|}
\hline $\mathbf{1}$ & $\mathbf{2}$ & Piston & $\mathbf{3}$ & $\mathbf{4}$ \\
\hline Feeding & $\begin{array}{c}\text { Fine } \\
\text { Tuning }\end{array}$ & $\begin{array}{c}\text { Feeding/Fine } \\
\text { Tuning }\end{array}$ & $\begin{array}{c}\text { Fine } \\
\text { Tuning }\end{array}$ & Feeding \\
\hline $\mathrm{m}^{3} * \mathrm{r}$ & $\mathrm{m} * \mathrm{r}$ & Stroke Length & $\mathrm{r}$ & $\mathrm{m}^{2} * \mathrm{r}$ \\
\hline
\end{tabular}

(B)

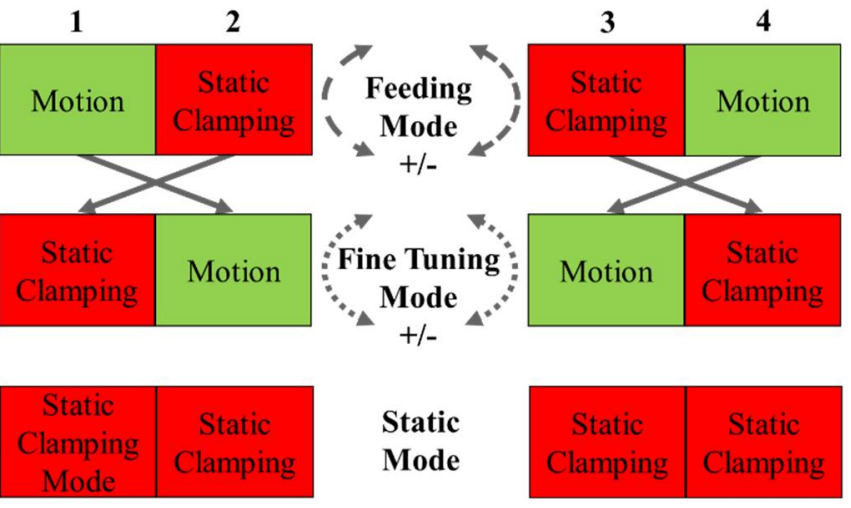

(C)

Figure 25. Incremental angular control in one DOF of two interconnected robot segments.

Fig. $25 \mathrm{C}$ shows the different main modes: Feeding Mode, Fine Tuning Mode and Static Mode. In Feeding Mode is piston 2 and 3 active and clamped to the piston rod, in the subsequent Fine Tuning Mode, piston 1 and 4.

The work sequence of the incremental angular control in one DOF of two interconnected robot segments is as follows. To begin with, piston 1 and 4 work with positioning left and right piston rod and actuation cables in the Fast Feeding Mode. The longer movements of the piston rods occurs here. Then there is a changeover to the Fine Tuning Mode and piston 2 and 3 position piston rods and actuation cables are moved until the commanded angle is obtained. It is important to note that the piston rods movements can occur in both directions.

An angular position sensor, not shown in figure 25A, closes the control loop including appropriate filters. The control loop will also include corrective commands in order to reach the commanded angle position. Finally in the Static Mode all pistons are clamped to the piston rod pair, enabling high stiffness.

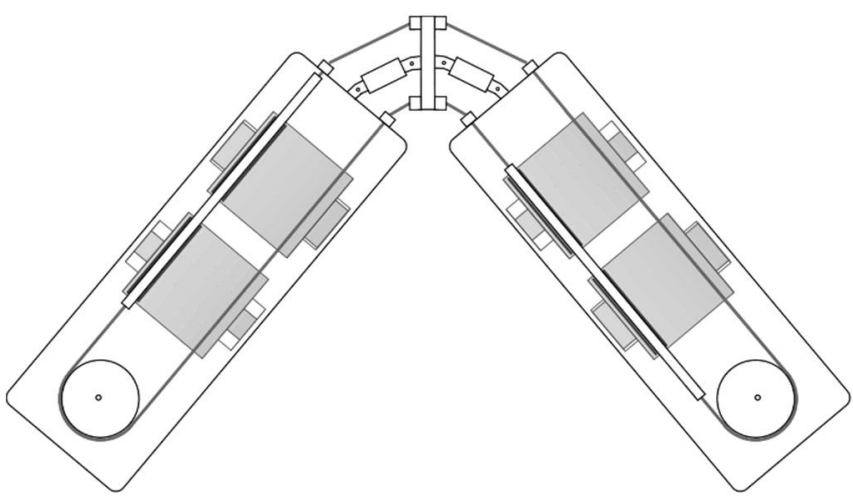

Figure 26. Example of rotational actuation enabling high agility.

Here it is showed that an incremental control strategy with a few large and small incremental steps available in four different pistons, can provide a powerful combination of high angle resolution and a large total angle stroke, enabling a high agility. The robot arm segments can be built together in series creating a snake-like robot capable of working in confined spaces and reach the farthest ends of the cavities of for example an aircraft while maintaining the rigidity and precision all the way to the end effector, see fig. 26 and 28. Also a combination with segments with HILA MR with incremental control and slave segments is a feasible solution.

\subsection{Need for slim snake-like robots}

Assembling modern aircrafts is, in many ways, still as much of a craft as 18th century shipbuilding, requiring loads of skill and manual labour to get the job done, because the needed industrial robots are too inflexible and reach too short [9]. There is a need for snake-like robots and lot of uses in several industrial segments today, beyond aircraft manufacturing, in automotive industry, in nuclear power plants, off-shore platforms, shipbuilding, laparoscopic surgery etc. But these robots are not on the market today, however there are some demonstrators.

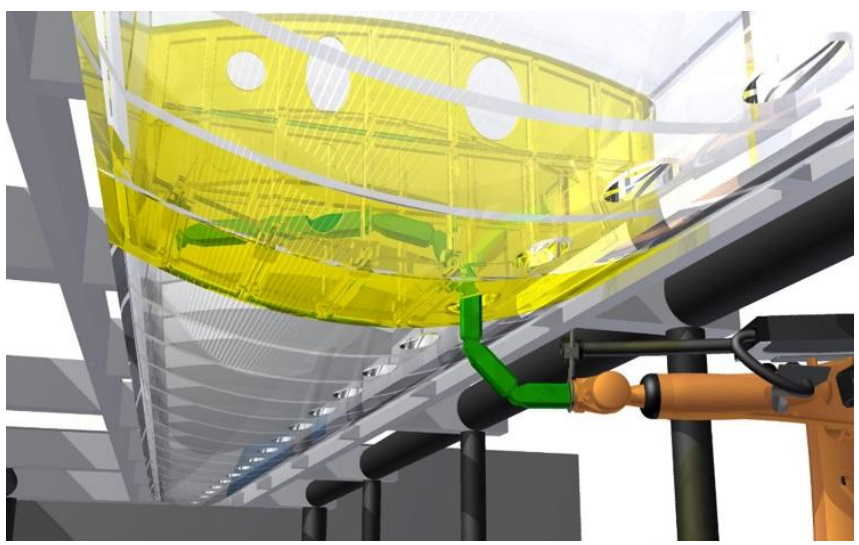

Figure 27. A snake-like robot in a wing tank (Image: FRAUNHOFER IWU).

Present articulated 6-, 7-axis electrical or hydraulic robots are too clumsy, especially in turning points, for this applications today. It requires distinct robotic arm movements performed 
by slim, multi joint robotic arms in tight spaces, avoiding to collide with walls in confined spaces, see fig. 27.

A large number of snake arm robot designs have been presented, but there is no snake robot that can carry a relatively large payload, while having small diameter/length ratio, great flexibility (bending capability) and a compact actuation system [10].

Robot arm segments, based on HILA MR with incremental control, can be designed as a snake-like robot, which consists of several series-connected elements. This robot is capable of working in confined spaces and reach the furthest ends of the cavities, for example, in an aircraft wing while maintaining the rigidity and precision all the way to the end effector. The snake-like HILA robot can be divided into a part that looks more like a jig, which is not operated so frequently, and a part near the end effector which is more like an industrial robot. HILA MR with incremental control can be used especially in parts which are less frequently operated and rapid movements are not so needed.

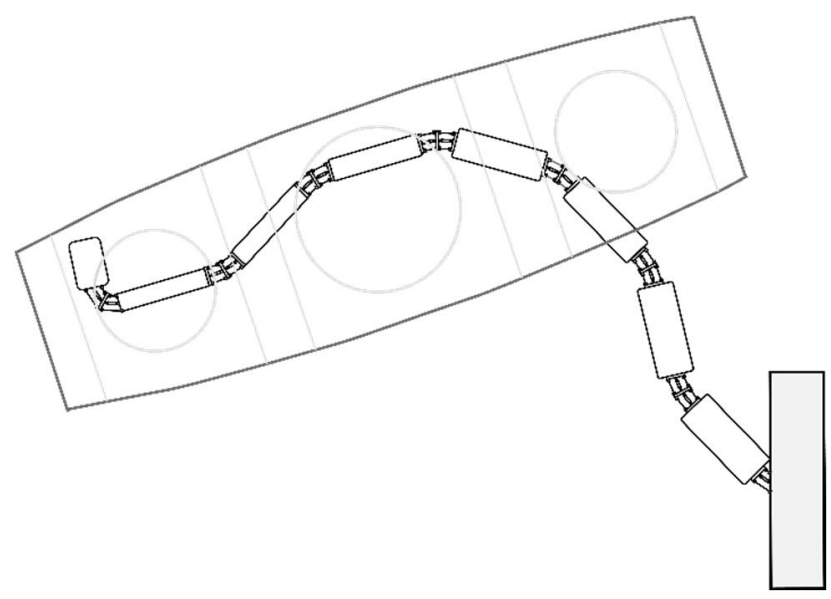

Figure 28. A snake-like HILA robot capable of working in confined spaces.

\section{Discussion}

HILA and its various combinations represents a quite different approach compared to conventional hydraulic cylinder technologies. The barrier is high to introduce new technology in the conservative hydraulic cylinder industry, mainly because established technology is so widely accepted. However, this industry needs new inventions to defend shrinking market segments [11].

Research has been carried out during several years in the field of digital hydraulic actuation. A number of promising concepts characterised by robustness, simplicity and reliability have been proposed. But commercial breakthrough has not yet been achieved, mainly due to the high number of needed on/off-valves and physical size and weight of the valve package.

HILA represents a new sort of digital hydraulics, especially HILA equipped with incremental control with discrete actuator stepping instead of switched valves. Various HILA technologies and concepts presented in this paper are based on few micro valves, which are working as a hydraulic transistor in each actuator. However, these high pressure and fast switching control valves require extremely low flow. HILA offers a miniaturisation of the control valve package and a neat design compared to present digital hydraulic systems.

\section{Future Work}

This conceptual paper presents a part of the current state of HILA development project. The basic functionality of HILA technology has successfully been proved in earlier model simulations and rig tests (see also https://m.youtube.com/watch?v=UCO-OQOdxtg). However, the work presented in this paper will be further enhanced by simulation and laboratory tests. Such future work may include that incremental control system needs to be modelled and simulated for different load and speed cases. The simulations will be done with and without mass inertia together with appropriate regulator structures, such as PID control, in order to minimise potential jerkiness and fatigue issues.

Another area of importance for further research work is finding suitable applications for proposed HILA technologies. This may include completely new applications for hydraulic cylinders in materials handling and mobile applications. All areas suitable for the variants of HILA described in this paper have not been identified for development efforts and research.

It is also important to identify industries that are in a dynamic development phase, and thus interested in new innovations in these areas. These may be found outside the traditional industries for hydraulic cylinders. To succeed in research with HILA, the technology also has to be presented to industries which today use electrical actuators to a large extent. Such an industry is the growing robot industry. HILA can offer the robot industry a more cost-effective and suitable technology for some applications, such as snakelike robots, where current electrical actuators do not fully meet the requirements for that application.

\section{Conclusions}

In this paper a number of concepts of HILA technology are presented. The basic invention is a hydraulic cylinder with a releasable piston. It is based on a well-known hydraulic clamping element used in a new combination, where the piston and the piston rod can quickly be coupled and uncoupled by means of the clamping element, in a failsafe way. The invention provides new features to hydraulic cylinders such as providing very long strokes, high rod speed, and small chamber volumes enabling high pressure which means high stiffness and low capacitance. The technology also represents a new sort of digital hydraulics, especially the variant with incremental control. The study presents feasible combinations of the technology.

HILA with incremental control, using ordinary directional valve and on/off valves, adds new features to present 
hydraulic cylinder technology and can be a potential alternative to expensive electric and hydraulic servo systems, when high position accuracy is the primary goal within less responsive closed loop systems.

HILA with multiple rods (HILA MR) technology is presented for the first time in this paper, enables a completely new and novel way to generate and distribute mechanical linear movement and force by using hydraulic actuators, maintaining high pressure, compactness and energy efficiency. A single position sensor on the common piston can be used to determine the absolute position of the individual piston rod.

In less responsive closed loop systems, HILA with multiple rods and incremental control enables a new way to generate and distribute mechanical linear movement and force by using hydraulic actuators in a cost effective way. Multiple actuating elements together with incremental control, integrated in a compact and lightweight single unit controlled by ordinary directional valves enables a high position accuracy.

In the robotic area a high agility robot configuration that may carry a reasonable load, with a small diameter and length ratio, is needed in several areas, such as nuclear power plants, off-shore platforms, shipbuilding, laparoscopic surgery etc. The robot has to be capable of working and navigating in confined spaces and reach the furthest ends of the cavities, for example in a wing tank, a collapsed building or in military operations.

HILA with multiple rods and incremental control enables a promising solution for these issues. With large and small incremental steps executed in two linked robot arm segments, it will be able to have a more accurate and time efficient method for angle positioning in pitch and yaw. The segments can be built together in series for snake-like robot applications.

HILA technology is patent pending.

\section{Acknowledgments}

This work was sponsored by Saab $\mathrm{AB}$ and the Swedish Energy Agency.

\section{References}

[1] K.-E. Rydberg,"Hydraulic servo systems", https://www.iei.liu.se/flumes/tmhp51/filearchive/course material/1.105708/HydServoSystems_part1.pdf, pp. 3, 2008

[2] M Landberg, M Hochwallner, and P Krus, "Novel Linear Hydraulic Actuator". ASME/BATH 2015 Symposium on Fluid Power \& Motion, Chicago, United States

[3] J Li, "Design and development of a piezoelectric linear actuator for smart structures", Department of Mechanical and Industrial Engineering, Concordia University, Montreal, Quebec, Canada, 2004

[4] M Hochwallner, M Landberg, and P Krus, "The Hydraulic Infinite Linear Actuator - properties relevant for control". In 10th International Fluid Power Conference (10. IFK), Vol. 3, pp. 411-424, 2016

[5] S Mraz, "Comparing Electric Rod Actuators and Hydraulic Cylinders", Machine Design, http://machinedesign.com/technologies/hydraulics, 2016

[6] M Linjama, "DIGITAL FLUID POWER -STATE OF THE ART", The 12th Scandinavian International Conference on Fluid Power, Tampere, Finland Linköping, 2011

[7] ETP-OCTOPUS, ETP Transmission AB, www.etptrans.se

[8] M Recksiek, "ADVANCED HIGH LIFT SYSTEM ARCHITECTURE WITH DISTRIBUTED ELECTRICAL FLAP ACTUATION", AST 2009, Hamburg, Germany

[9] "Automated assembly of aircraft wings" , https://www.fraunhofer.de/en/press/researchnews/2014/may/automated-assembly-of-aircraftwings.html

[10] X Dong, M Raffles, S Cobos Guzman, D Axinte, J Kell, "Design and analysis of a family of snake arm robots connected by compliant joints", Mechanism and Machine Theory 77 (2014) 73-91

[11] "Hydraulic Cylinders Industry Report", July 2013, InterOcean Advisors LLC, 117 North Jefferson Street, Suite 205, Chicago, IL 60661, www.ioadvisors.com 